\title{
Correction to: The Barcelona ionospheric mapping function (BIMF) and its application to northern mid-latitudes
}

\author{
Haixia Lyu ${ }^{1,2} \mathbb{( D} \cdot$ Manuel Hernández-Pajares ${ }^{1,3}\left(\mathbb{D} \cdot\right.$ Metin Nohutcu $^{4} \cdot$ Alberto García-Rigo $^{1,3}$ (D) Hongping Zhang ${ }^{2}$. \\ Jingnan Liu ${ }^{2}$
}

Published online: 20 July 2020

(c) Springer-Verlag GmbH Germany, part of Springer Nature 2020

\section{Correction to: GPS Solutions (2018) 22:67 https://doi.org/10.1007/s10291-018-0731-0}

In the original article, some typos regarding Eq. (3) and Table 9 are introduced.

Corrections to Eq. (3)

$$
\begin{aligned}
\text { STEC } & =N_{1} l_{1}+N_{2}^{\prime} l_{2}^{\prime} \\
& =H \cdot \frac{N_{1} l_{1}}{H}+H \cdot \frac{N_{2}^{\prime} l_{2}^{\prime}}{H} \\
& =P_{1} \cdot M_{1}+P_{2}^{\prime} \cdot M_{2}^{\prime} \\
& =M_{1} \cdot \mu_{1} V+M_{2}^{\prime} \cdot \mu_{2}^{\prime} V^{\prime} \\
& =\left(1-\mu_{2}\right) M_{1} V+\mu_{2}^{\prime} M_{2}^{\prime} V^{\prime}
\end{aligned}
$$

The original article can be found online at https://doi.org/10.1007/ s10291-018-0731-0.

Manuel Hernández-Pajares

manuel.hernandez@upc.edu

Haixia Lyu

haixialv1987@gmail.com

Metin Nohutcu

mnohutcu@ hacettepe.edu.tr

Alberto García-Rigo

alberto.garcia.rigo@upc.edu

Hongping Zhang

hpzhang@whu.edu.cn

Jingnan Liu

jnliu@whu.edu.cn

1 UPC-IonSAT, Universitat Politècnica de Catalunya,

Barcelona, Spain

2 GNSS Research Center, Wuhan University, Wuhan, China

3 IEEC-CTE-CRAE, Institut d'Estudis Espacials de Catalunya, Barcelona, Spain

4 Department of Geomatics Engineering, Hacettepe University, Ankara, Turkey
$V$ and $V^{\prime}$ are VTECs for the whole ionosphere at IPP1 and IPP2, respectively; $\mu_{2}$ and $\mu_{2}^{\prime}$ are the shape functions of the voxels for the second-layer corresponding to IPP1 and IPP2; $M_{1}$ and $M_{2}^{\prime}$ are the standard mapping functions at IPP1 and IPP2, equal to $l_{1} / H$ and $l_{2}^{\prime} / H$ respectively.

The wrong table references in Section "Assessment" are corrected as below:

"It is worth mentioning that the number of available days is less than 365, as shown in Tables 1, 8 and 9 in Appendix $\mathrm{B}$, because the days in which the selected station was used in the GIMs computation are not considered for a fair assessment."

"As shown in Table 9, the BIMF improved dSTEC using JPLG GIMs as well, with an improvement given by \%dB in more than $70 \%$ of cases."

Publisher's Note Springer Nature remains neutral with regard to jurisdictional claims in published maps and institutional affiliations. 